\title{
ANÁLISE DA MELHORA DE DOR EM PACIENTES COM LOMBALGIA SUBMETIDOS A PROCEDIMENTOS FISIOTERAPÊUTICOS
}

\author{
Bruna Schwingel Albrecht ${ }^{1}$ \\ Cassia da Luz Goulart ${ }^{1}$ \\ Luciana Cezimbra Weis ${ }^{2}$
}

\begin{abstract}
RESUMO
A lombalgia é definida como um quadro doloroso na região lombar afetando cerca de $70 \%$ da população em que se busca analgesia através de estimulação elétrica nervosa transcutânea (TENS). Neste sentido se objetivou analisar o efeito do atendimento fisioterapêutico em pacientes com lombalgia. Estudo de caráter clínico não randomizado realizado na Clínica FisioUNISC, no projeto de pesquisa intervenções terapêuticas nas alterações músculo esqueléticas em pacientes com disfunções da coluna vertebral. Sendo avaliados 14 pacientes portadores de lombalgia clinicamente diagnosticada, submetidos à ficha de avaliação fisioterapêutica, escala visual analógica (EVA), goniometria e avaliação postural, bem como de um questionário de qualidade das atividades de vida diária (AVD's). A média de idade foi $55,79 \pm 14,21$ anos e predomínio do sexo feminino $(71,4 \%)$, os dados foram coletados antes e após sessões de intervenção fisioterapêutica, por meio do TENS. Os resultados apontam melhora no nível de dor avaliada pela EVA quando comparados pré e pós atendimento $(5,29 \pm 0,65$ vs $3,36 \pm 0,37)$, respectivamente $(p=0,005)$. O sexo masculino destacou-se pela redução da dor quando comparados o pré atendimento com o pós atendimento $(7,25 \pm 1,03 \mathrm{vs}$ $3,75 \pm 0,62)$. Através dos métodos empregados observa-se uma redução significativa de dor do pré para o pós atendimento.
\end{abstract}

Palavras-chave: Lombalgia. TENS. Escala visual analógica (EVA). Dor.

\begin{abstract}
Low back pain is defined as a painful condition in the lower back affecting about $70 \%$ of the population it seeks to analgesia through transcutaneous electrical nerve stimulation (TENS). In this sense it aimed to analyze the effect of physical therapy in patients with low back pain. Clinical character of non-randomized study conducted at the Clinic FisioUNISC in the research project therapeutic interventions on musculoskeletal disorders in patients with disorders of the spine. Being evaluated 14 patients with low back pain clinically diagnosed, undergoing physical therapy assessment form, visual analogue scale (VAS), goniometer and postural evaluation and a quality survey of the activities of daily living (ADLs). The mean age was $55.79 \pm 14.21$ years and a predominance of females $(71.4 \%)$, data were collected before and after physical therapy intervention sessions, through the TENS. The results show improvement in the level of pain assessed by VAS when compared before and after treatment $(5.29 \pm 0.65$ vs $3.36 \pm 0.37)$, respectively $(\mathrm{p}=0.005)$. The men stood out for pain reduction, from before to after care service $(7.25 \pm 1.03$ vs $3.75 \pm 0.62)$. Through the methods used there has been a significant reduction of pain pre and post care.
\end{abstract}

Keywords: Low back pain. TENS. Visual analogue scale (VAS). Pain.

1 Alunas do Curso de Fisioterapia da Universidade de Santa Cruz do Sul - UNISC. $\leq$ bru_albrecht@yahoo.com.br $\geq$ e $\leq$ luz.cassia@ hotmail.com $\geq$

2 Professora do Departamento de Educação Física e Saúde (DEDFIS) na Universidade de Santa Cruz do Sul UNISC. $\leq$ weis@unisc.br $\geq$ 


\section{INTRODUÇÃO}

A dor é definida como uma experiência sensorial e emocional desagradável associada a um dano real ou potencial dos tecidos (LIMA et al., 2011). Neste sentido, a dor lombar é considerada um dos principais problemas de saúde pública, sendo que nesta está inclusa a lombalgia, que é uma síndrome decorrente do uso excessivo, da atuação de forças compressivas ou alterações posturais, relacionada à fraqueza e desequilíbrios musculares. Diminuindo assim a amplitude ou a coordenação de movimentos, aumentando a fadiga e a instabilidade de tronco, associando-se também a fatores psicológicos que acabam afetando a qualidade de vida (SILVA et al., 2014).

A avaliação destes pacientes que possuem alterações lombares é composta de dados funcionais, índices de dor como a Escala Visual Analógica (EVA), avaliação postural, questionários de qualidade de vida, exames médicos (FELLET et al., 2010). A EVA é um instrumento de suma importância e sua aplicabilidade possibilita a tomada de decisões importantes no que se refere à dor do paciente e ainda nos proporciona, com maior exatidão, quantificar a dor que está sentindo e se a terapêutica que está sendo utilizada realmente está sendo eficaz no tratamento (BOTTEGA; FONTANA, 2010).

Utilizam-se, então, algumas formas de tratamento das lombalgias é através de recursos físicos, como a estimulação elétrica nervosa transcutânea (TENS), que com suas prerrogativas e pesquisas demonstram resultados promissores que podem reabilitar e/ou prevenir crises frequentes e recorrentes de dor e limitações funcionais (PIRES; DUMAS, 2008). Segundo Johnson (2003) e Agne (2004), citado por Gauglitz (2005) é uma técnica analgésica e não invasiva utilizada para aliviar os sintomas de dor aguda e crônica. Essa modalidade pode ser dividida em Convencional, Breve Intenso, Burst e Acupuntura, com diferentes tempos de analgesia para os pacientes.

Outro recurso na prevenção de dores e do cansaço físico e mental, a massoterapia tem a finalidade de produzir efeitos sobre os sistemas nervoso, muscular e respiratório e na circulação local e geral do sangue e da linfa (BORGES; KUREBAYASHI; SILVA, 2014). Ainda pode se associar com o emprego da cinesioterapia, que, através de alongamentos utilizados principalmente para ganho de flexibilidade das fibras musculares que estejam encurtadas, busca recuperar a extensibilidade dos ligamentos e tendões, o que melhora a amplitude de movimento, diminui o estresse e a tensão e aumenta o fluxo sanguíneo, 
diminuindo consequentemente a dor (GODINHO et al., 2011). Neste sentido se objetivou analisar o efeito do atendimento fisioterapêutico em pacientes com lombalgia.

\section{MATERIAIS E MÉTODOS}

O estudo segue um modelo de estudo clínico não randomizado (GOLDIM, 2000) que ocorreu no período de março a julho de 2014 na Clínica Escola do Curso de Fisioterapia da UNISC - FisioUnisc, em Santa Cruz do Sul - RS, junto ao Projeto de Pesquisa Intervenções terapêuticas nas alterações músculo esqueléticas em pacientes com disfunções da coluna vertebral. O projeto de pesquisa foi submetido ao CEP da UNISC para apreciação ética em pesquisa com seres humanos, de acordo com a Resolução CNS n 466/12, e aprovado sob designações do CAAE 32010314.6.0000.5343 e $\mathrm{n}^{\mathrm{o}}$ de parecer 726.008. Os sujeitos foram convidados a participarem desta pesquisa, onde inicialmente foi lido o Termo de Consentimento Livre e Esclarecido - TCLE assinado em duas vias, permanecendo uma com o voluntário e outra com as pesquisadoras.

Os critérios de inclusão foram sujeitos adultos e/ou idoso do sexo masculino e feminino, com diagnóstico clínico comprobatório de lombalgia, com quadro doloroso lombar, quer seja em fase aguda ou crônica, com sensibilidade da região lombar preservada, limitações de movimentos das regiões nas atividades cotidianas e/ou com alterações posturais e tendo ou não realizado tratamento de fisioterapia a mais de um ano. Já os critérios de exclusão foram sujeitos que não aceitassem assinar o TCLE, com presença de contra indicações ao uso dos recursos terapêuticos como: alergia local, falta de sensibilidade local, hipertensos descompensados, cardiopatas severos, processos cancerosos malignos, implantes metálicos em área lombar e exacerbação dolorosa aos processos terapêuticos empregados na pesquisa.

Inicialmente esses sujeitos foram recepcionados junto ao projeto, onde passaram por um processo de anamnese realizado pelo acadêmico bolsista do projeto e acompanhado por profissional fisioterapeuta composto de ficha de avaliação fisioterapêutica, contendo sinais vitais, EVA, sensibilidade tátil com uso de estesiômetros e sensibilidade térmica com bolsa gel quente e fria, goniometria e testes de força muscular da região lombar. Avaliação postural e questionário de qualidade de satisfação das AVD's, adaptado para versão brasileira do Swiss spinal stenosis questionnaire (AZUAGA et al., 2013). Essa avaliação foi realizada antes de iniciar o tratamento e no final do tratamento fisioterapêutico. Antes de cada sessão eram 
aferidos os sinais vitais, como pressão arterial (PA), saturação periférica de oxigênio $\left(\mathrm{SpO}_{2}\right)$, frequência cardíaca (FC) e respiratória (FR). Em seguida, os pacientes foram questionados quanto à presença de dor naquele momento antes da intervenção através da EVA, quando se explicava que 0 era "sem dor" e 10 a "dor mais insuportável” que o paciente já sentiu. Assim que terminava a sessão de fisioterapia era novamente questionada a dor e explicado que eles deveriam relatar a dor que estavam sentindo no momento, não podendo omitir nenhuma informação, e após eram aferidos os sinais vitais.

Cada sessão de intervenção durava 50 minutos, sendo aplicada Estimulação Elétrica Nervosa Transcutânea (TENS), no equipamento marca quark®, modelo TENSVif 993, modo acupuntura $(\mathrm{T}=80 \mu \mathrm{s} ; \mathrm{F}=20 \mathrm{~Hz})$ aplicados com 4 eletrodos grandes de borracha silicone carbono, distribuídos de forma bilateral, horizontal e longitudinal na região lombar, onde o recurso eletroterápico foi empregado por 20 minutos (AGNE, 2013). Associou-se ainda o recurso de massoterapia através de técnicas de deslizamento superficial, profundo e fricção que são mais relaxantes para musculatura por 10 minutos (ABAD et al., 2010) e cinesioterapia com alongamentos simples da musculatura lombar bilateral compondo-se de séries de 10 movimentos intercalados por um intervalo de 30 segundos entre as mesmas, durante os últimos 20 minutos da sessão fisioterapêutica (KISNER; COLBY, 2009; RIBEIRO; MOREIRA, 2010).

Os resultados da EVA pré e pós intervenção fisioterapêutica, enfatizados neste artigo, foram analisados pelo programa Statistical Package for Social Sciences for Windows (SPSS, versão 20.0) com a estatística expressa em valores percentuais, médias e desviospadrão e comparados pelo teste de Wilcoxon $(\mathrm{p}<0,05)$.

\section{RESULTADOS E DISCUSSÃO}

Em triagem inicial foram avaliados 23 pacientes, dos quais 14 foram diagnosticados com laudos clínicos comprobatórios de dor lombar caracterizada como lombalgia, sendo estes incluídos no presente estudo. Na Tabela 1 podem ser observadas as características e os sinais vitais dos sujeitos deste estudo. 
Tabela 1 - Caracterização da amostra e sinais vitais

\begin{tabular}{cc}
\hline Variáveis & $\mathrm{n}=14$ \\
\hline Sexo, feminino & $10(71,4 \%)$ \\
Sexo, masculino & $04(28,6 \%)$ \\
Idade (anos) & $55,79 \pm 14,21$ \\
Qualidade de Vida & $26,50 \pm 5,90$ \\
$\mathrm{PAS}(\mathrm{mmHg})$ & $130,07 \pm 11,12$ \\
$\mathrm{PAD}(\mathrm{mmHg})$ & $80,70 \pm 9,90$ \\
$\mathrm{FC}(\mathrm{bpm})$ & $74,30 \pm 13,40$ \\
$\mathrm{SPO}(\%)$ & $97,14 \pm 1,80$ \\
$\mathrm{FR}($ irpm$)$ & $16 \pm 2,30$ \\
\hline Dados expressos em frequência, média e desvio padrão. PAS: pressão arterial sistólica; PAD: pressão arterial diastólica; FC: \\
frequência cardíaca; $\mathrm{SPO}_{2}$ : saturação periférica de oxigênio; FR: frequência respiratória.
\end{tabular}

A avaliação destes pacientes que possuem alterações lombares é composta de dados funcionais, índices de dor através de escalas como a Escala Visual Analógica (EVA), avaliação postural, pesquisas de qualidade de vida, exames médicos, exames eletromiográficos (análise de força e/ou fadiga muscular), além da possibilidade de avaliação da influência bioquímica na dor. Esclarecer as devidas situações decorrentes do processo sintomatológico dos pacientes com dor lombar é importante para podermos propor tratamentos corretos e mais adequados a cada situação problema nestes indivíduos. Desde exames simples de raio $\mathrm{x}$, a outros mais aprimorados como as ressonâncias magnéticas e/ou tomografias computadorizadas, eletromiografia, favorecerão a se definir com maior credibilidade se o problema advém de ordem óssea, muscular, ligamentar ou tendínea. Muitas vezes é o somatório de várias destas circunstâncias ao mesmo tempo (FELLET et. al., 2010).

Conforme HELFENSTEIN et al. (2010), o número de pessoas com lombalgia é considerado um problema de ordem crescente, atingindo de $50 \%$ a $80 \%$ dos atendimentos na área da saúde, entretanto destaca-se que as mulheres sejam as mais predispostas a esta patologia, dados estes que corroboram com os resultados deste estudo.

A dor é um fenômeno multidimensional, tendo componentes sensitivos, emocionais e cognitivos. Dependendo da sua intensidade e frequência pode gerar desconfortos físicos e psicológicos, dificultando o desenvolvimento das atividades cotidianas e da qualidade de vida. A prevalência da dor lombar é reportada em todo o mundo, porém não há um consenso a respeito da sua etiologia e na maioria dos casos é classificada como de origem "não específica". Estudos mostram vários fatores relacionados como o desenvolvimento da dor lombar, como a interação social negativa e problemas de origem mecânica. Associada a essa dor, observa-se uma redução temporária nas atividades de vida diárias, podendo exercer 
efeitos sobre os sistemas cardiovascular e musculoesquelético, o que acaba potencializando as perdas funcionais (FERREIRA et al., 2010; MONTENEGRO et al., 2010).

A lombalgia é responsável por uma grande parte das faltas no trabalho, sendo estimada por estudos entre os 5 a 10 diagnósticos mais frequentes, permanecendo estável ao longo dos anos (HELFENSTEIN et al., 2010). Há vários métodos para quantificar a dor, um deles é a Escala Visual Analógica da dor (EVA). É constituída por uma barra horizontal em forma de régua, com uma escala de 0 a 10, onde o individuo deve informar a intensidade de sua dor, na qual 0 é sem dor e 10 a dor mais intensa/máxima sentida pelo indivíduo/paciente (PEREIRA et al., 2011).

Partindo-se da condição e do pressuposto de uma boa avaliação das principais alterações decorrentes da lombalgia, podemos destacar o uso de recursos físicos de tratamento, através da eletroterapia, massoterapia e cinesioterapia. O termo eletroterapia consiste na aplicação da corrente elétrica com finalidade terapêutica, baseia-se na aplicação de energia eletromagnética ao organismo com finalidade de produzir sobre ele reações fisiológicas, terapêuticas e também de investigação. A sigla TENS significa Estimulação Elétrica Nervosa Transcutânea, que por sua vez consiste na aplicação de eletrodos sobre a pele com o objetivo de estimular as fibras nervosas grossas A-Alfa (sensoriais) mielinizadas de condução rápidas, como por exemplo, as fibras motoras. Esta estimulação desencadeia a nível central os sistemas analgésicos, gerando a redução da dor (AGNE, 2013).

O TENS acupuntura tem a duração do impulso elevado em torno de 180 até $250 \mu$ s e a frequência sempre abaixo de $10 \mathrm{~Hz}$. A intensidade deve ser elevada até o indivíduo sentir leves contrações e/ou fibrilações musculares. Esse processo estimula a liberação de Bendorfina e promove o relaxamento das fibras musculares, retirando as toxinas e melhorando o metabolismo local (AGNE, 2009). Esse tipo de TENS tenta minimizar o efeito mecânico promovido pela introdução da agulha nos pontos acupunturais e o indivíduo deve sentir uma sensação de parestesia adicionada a abalos musculares como citado acima. (MONTENEGRO et al., 2010). Devido o TENS melhorar a dor, consequentemente, ocorre a melhora dos sintomas secundários, como os distúrbios do sono, condições emocionais e qualidade de vida relacionada à saúde (SILVA et al., 2008).

A massagem é benéfica para a lombalgia que não for proveniente de doenças, seja subaguda ou crônica, e também apresenta efeitos a longo prazo após o término do tratamento. Outros efeitos da massagem são o alívio de dores, tensões musculares e relaxamento, promovendo sensação de bem estar (BORGES et al., 2012). 
Na Fisioterapia, a cinesioterapia é uma das linhas de escolha de tratamento para auxiliar na lombalgia, sendo o alongamento, especialmente o estático, muito utilizado (PUPPIN et al., 2011). O alongamento muscular é muito eficaz no tratamento das mais diversas disfunções do sistema músculo esquelético, sendo uma das melhores técnicas para aumento da flexibilidade. O alongamento é uma manobra terapêutica empregada para aumentar o comprimento de tecidos moles que estão encurtados, e pode ser definida também como técnica aplicada para aumentar a extensibilidade músculo tendínea e do tecido conjuntivo periarticular, contribuindo para aumentar a flexibilidade articular (MACHADO et al., 2010).

Considerando os dados da EVA pré e pós atendimento fisioterapêutico, pode-se observar que, de uma forma geral, houve uma melhora estatisticamente significativa na dor pós atendimento $(p=0,005)$. Na Tabela 2 , quando analisado a redução da dor por sexo, os homens apresentaram melhoras evidentes do pré atendimento para o pós atendimento $(7,25 \pm 1,03$ vs $3,75 \pm 0,62, p=0,001)$.

Tabela 2 - Demonstrativo do índice de dor pré e pós atendimento

\begin{tabular}{cccc}
\hline \multicolumn{2}{c}{ Variáveis } & $\mathrm{n}=14$ & Valor de $p$ \\
\hline & Pré Atendimento & Pós Atendimento & 0,005 \\
EVA & $5,29 \pm 0,65$ & $3,36 \pm 0,37$ & 0,080 \\
EVA, sexo feminino & $4,50 \pm 0,68$ & $3,20 \pm 0,46$ & 0,001 \\
\hline EVA, sexo masculino & $7,25 \pm 1,03$ & $3,75 \pm 0,62$ &
\end{tabular}

EVA: Escala Visual Analógica

Estudos relatam que a EVA é importante no sentido de acompanhar a evolução da intensificação da dor, se o tratamento está beneficiando o paciente e se a evolução doença se torna mais aguda (BOTTEGA; FONTANA 2010). Já em um estudo realizado por Lima et al. 2011, cita que a classificação da dor nos pacientes teriam um grau de dor moderada, no entanto após realizarem TENS, obtiveram uma redução do grau de dor. Resultado que vem ao encontro do presente estudo, uma vez que os pacientes reduziram significativamente o grau de dor.

Por outro lado, a maior redução de dor, apresentada pelo sexo masculino neste estudo pode ser explicada pela carga hormonal destes, porém há poucos estudos justificando este resultado. Já que são mais frequentes as pessoas do sexo feminino obter uma redução de dor mais rápida e significativa, entretanto torna-se subjetivo uma vez que há vários fatores 
envolvidos, desde cargas mecânicas, fatores fisiológicos e intensidade da dor (FURTADO et al., 2014).

\section{CONCLUSÃO}

A partir dos dados obtidos por este estudo, pode-se concluir que as sessões de intervenção fisioterapêutica, empregadas nos atendimentos com pacientes que apresentavam lombalgias, foram de redução significativa na dor, quando comparado pré e pós tratamento. Estes achados indicam o possível benefício das técnicas fisioterapêuticas na melhora físicofuncional, na qualidade de vida e nas rotinas de vida diária das pessoas acometidas por lombalgia, ressaltando o papel da fisioterapia na redução da dor, respeitando a natureza individual de resposta aos tratamentos propostos.

\section{REFERÊNCIAS}

AGNE, J.E. Eu sei eletroterapia. Santa Maria: Pallotti, 2009.

AGNE, J.E. Eletrotermofototerapia. $2^{\text {a }}$ ed. Santa Maria: Pallotti, 2013.

AZUAGA, T.L. et al. Tradução e adaptação transcultural para língua portuguesa do "Swiss Spinal Stenosis Questionnaire”. Coluna/Columna. Campinas, n.1, v.12, p. 36-41, 2013.

BORGES, T.P., KUREBAYASHI, L. F. S., SILVA, M. J. P. Lombalgia ocupacional em trabalhadores de enfermagem: massagem versus dor. Revista da Escola de Enfermagem da USP. São Paulo, n.4, v.48, p.675-699, ago. 2014.

BORGES, L.T.P. et al. Aplicação da massagem para lombalgia ocupacional em funcionários de enfermagem. Revista Latino-Americana de Enfermagem. Ribeirão Preto, n. 3, v.20, p.511519, maio/jun. 2012.

BOTTEGA, F.H.; FONTANA, R.T.A dor como quinto sinal vital: utilização da escala de avaliação por enfermeiros de um hospital geral. Texto Contexto Enfermagem, n.2, v. 19, p. 283-290, abr./jun. 2010.

FELLET, A. J. et al. Lombalgias. Revista Brasileira de Medicina, Juiz de Fora, n.10, v. 57, p.03-11, out. 2010 .

FERREIRA, C.B. et al. Os efeitos de dois métodos de intervenção fisioterapêutica sobre a atividade postural estática de indivíduos com dor lombar crônica. Revista Brasileira de Ciências da Saúde, São Caetano do Sul - USCS, n.26, v. 8, p. 34-42, out./dez. 2010. 
FURTADO, R.N.V. et al., Dor lombar inespecífica em adultos jovens: fatores de risco associados. Revista Brasileira de Reumatologia, n.5, v.4, p.371-377, jul. 2014.

GAUGLITZ, A.G.C. et al. Comparação do grau de agradabilidade na aplicação da estimulação elétrica nervosa transcutânea e da corrente interferencial. Revista Brasileira de Ciências da Saúde, São Caetano do Sul, n. 6, p. 18-23, jul./dez. 2005.

JUNIOR, M.H.; GOLDENFUM, M. A.; SIENA, C. Lombalgia ocupacional. Revista da Associação Médica Brasiileira, São Paulo, n.56, v.5, p.583-589, maio 2010.

KISNER, C., COLBY, L. A. Exercícios terapêuticos: fundamentos e técnicas. 4. ed. São Paulo: Manole, 2005.

LIMA, P.M.B. et al., Estimulação elétrica nervosa transcutânea após cirurgia de revascularização miocárdica. Revista Brasileira de Cirurgia Cardiovascular, n.4, v.26, p. 591-596, out./dez. 2011.

MACHADO, G.F.; BIGOLIN, S. E. Estudo comparativo de casos entre a mobilização neural e um programa de alongamento muscular em lombálgicos crônicos. Fisioterapia em Movimento, Curitiba, n.4, v. 23, p. 545-554, out./dez. 2010.

MONTENEGRO, E.J.N. et al. Ação da TENS acupuntural em acupontos na dor induzida pela hipotermia local $\left(0-2^{\circ}\right.$ C). Fisioterapia em Movimento, Curitiba, n.3, v. 23, p. 483-492, jul./set. 2010.

PEREIRA, G.D. et al. Efeito da Corrente Interferencial, 2000Hz, no Limiar de Dor Induzida. Revista Brasileira de Medicina do Esporte, São Paulo, n.4, v. 17, p.257-260, jul./ago. 2011.

PIVETTA, K.M.; BERTOLIN, G. R. F. Efeitos do $\Delta \mathrm{F}$ sobre a acomodação da corrente interferencial em sujeitos saudáveis. Revista Brasileira de Medicina do Esporte, São Paulo, n.5, v. 18, p. 330-332, set./out. 2012.

PUPPIN, M.A.F.L. et al. Alongamento muscular na dor lombar crônica inespecífica: uma estratégia do método GDS. Fisioterapia e Pesquisa, São Paulo, n.2, v.18, p. 116-121, abr./jun. 2011.

RIBEIRO, I.Q.B. et al. Fatores ocupacionais associados à dor musculoesquelética em professores. Revista Baiana de Saúde Pública, Salvador, n.1, v.35, p.42-64, jan./mar. 2011.

RIBEIRO, C. MOREIRA, D. O exercício terapêutico no tratamento da lombalgia crônica: uma revisão de literatura. Revista Brasileira de Ciência e Movimento, São Paulo, n.4, v.18, p. 100-104, mar. 2010.

SILVA, J.C.A et al. Desempenho funcional e percepção da dor na lombalgia crônica após aplicação de um programa de back school, SANARE-Revista de Políticas Públicas, Sobral, n.1, v.13, p.18-22, jan./jun. 2014.

SILVA, T.F.G. et al. Comparação dos efeitos da estimulação elétrica nervosa transcutânea e da hidroterapia na dor, flexibilidade e qualidade de vida de pacientes com fibromialgia. Fisioterapia e Pesquisa, São Paulo, n.2, v.15, p. 118-124, abr. 2008. 
SOUZA, A.V.R. et al. Nível de atividade física e lombalgia entre funcionários de uma instituição de ensino superior no nordeste do Brasil. Revista Brasileira em Promoção da Saúde, Fortaleza, n.24, v.3, p.199-206, jul./set. 2011. 